\title{
Validation of the Body Dysmorphic Disorder Questionnaire in a community sample of Swedish women
}

\author{
Sabina Brohede, Gun Wingren, Barbro Wijma and Klaas Wijma
}

\section{Linköping University Post Print}

\section{Tweet}

N.B.: When citing this work, cite the original article.

Original Publication:

Sabina Brohede, Gun Wingren, Barbro Wijma and Klaas Wijma, Validation of the Body Dysmorphic Disorder Questionnaire in a community sample of Swedish women, 2013, Psychiatry Research, (210), 2, 647-652. http://dx.doi.org/10.1016/j.psychres.2013.07.019

Copyright: Elsevier http://www.elsevier.com/

Postprint available at: Linköping University Electronic Press http://urn.kb.se/resolve?urn=urn:nbn:se:liu:diva-103301 


\section{Validation of the Body Dysmorphic Disorder Questionnaire in a community sample of Swedish women}

Sabina Brohede ${ }^{a}$, Gun Wingren ${ }^{c}$, Barbro Wijma $^{d}$, Klaas Wijma $^{b}$

a Unit of Medical Psychology, Department of Clinical and Experimental Medicine, Faculty of Health Sciences, Linköping University and Department of Paediatrics, County Council of Östergötland, Linköping, Sweden.

b Unit of Medical Psychology, Department of Clinical and Experimental Medicine, Faculty of Health Sciences, Linköping University, Linköping, Sweden.

c Occupational and Environmental Medicine, Department of Clinical and Experimental Medicine, Faculty of Health Sciences, Linköping University, Linköping, Sweden.

d Gender and Medicine, Department of Clinical and Experimental Medicine, Faculty of Health Sciences, Linköping University, Linköping, Sweden.

*Corresponding author:

Sabina Brohede, M.D.

Unit of Medical Psychology

Department of Clinical and Experimental Medicine

Faculty of Health Sciences

University Hospital

S-581 83 Linköping

Sweden

Tel: +46702991428

Tel: +46101038931

E-mail: Sabina.Brohede@liu.se 


\section{Abstract}

Body Dysmorphic Disorder (BDD) is characterized by a distressing and impairing preoccupation with a nonexistent or slight defect in appearance. Patients with the disorder present to both psychiatric and non-psychiatric physicians. A few studies have assessed BDD prevalence in the general population and have shown that the disorder is relatively common. To date, no BDD assessment instruments have been validated in the general population. Our aim was to validate a brief self-screening instrument, the Body Dysmorphic Disorder Questionnaire (BDDQ), in a female community sample. The BDDQ was translated into Swedish and filled out by 2891 women from a randomly selected community sample. The questionnaire was validated in a subsample of 88 women, using the Structured Clinical Interview for DSM-IV (SCID) together with clinical assessment as the gold standard. In the validation subsample, the BDDQ showed good concurrent validity, with a sensitivity of $94 \%$, a specificity of $90 \%$ and a likelihood ratio of 9.4 . The questionnaire can therefore be of value when screening for BDD in female populations.

\section{Key words}

Self-report instrument; Measurement; Somatoform disorders; Appearance concerns; Body image 


\section{Introduction}

Body Dysmorphic Disorder (BDD) is a psychiatric disorder, defined in the Diagnostic and Statistical Manual of Mental Disorders (DSM)-IV as a distressing and impairing preoccupation with an imagined defect in appearance; if a slight physical anomaly is present, the person's concern is markedly excessive (American Psychiatric Association, 2000). BDD is characterized by a pattern of obsessive thoughts, feelings and compulsive behaviors. The preoccupations are very time consuming (occurring on average 3-8 hours a day) and usually difficult to resist or control (Phillips and Hollander, 2008). BDD is associated with significant distress, disability (including social isolation and occupational dysfunction), cosmetic surgery and suicidality (Phillips, 1991; Crerand et al., 2005; Phillips, 2007). BDD appears to be relatively common. The three largest studies $(n>2000)$ in general populations found BDD prevalence rates of 1.7-2.4\% (Rief et al., 2006; Koran et al., 2008; Buhlmann et al., 2010). Using structured clinical interviews examining community samples, prevalence rates of $0.7-3 \%$ have been reported (Faravelli et al., 1997; Bienvenu et al., 2000; Otto et al., 2001). Studies of psychiatric samples have reported BDD in 2.6-16.0\% of patients (Zimmerman and Mattia, 1998; Grant et al., 2001; Conroy et al.; 2008, Kollei et al., 2011,). In dermatology settings, most studies have found BDD rates of 8.8-14\% (Phillips et al., 2000; Uzun et al., 2003; Bowe et al., 2007). The ratio of females to males is in the range of $1: 1$ to $3: 2$ (Phillips et al., 2008).

The Body Dysmorphic Disorder Questionnaire (BDDQ) is a brief, self-report measure, which is derived from the DSM-IV diagnostic criteria for BDD. Using close-ended questions it asks the respondents whether their appearance concerns are sources of preoccupation and, if so, it assesses the degree to which they cause distress or interfere with the person's social or occupational functioning (Phillips, 2009). The questionnaire was developed as a screening instrument for BDD in psychiatric settings and was validated in a psychiatric outpatient sample $(n=66)$, displaying high sensitivity $(100 \%)$ and specificity $(89 \%)$ (Phillips et al., 1995). In a psychiatric inpatient sample $(n=122)$ the sensitivity was $100 \%$ and the specificity was 93\% (Grant et al., 2001). A slightly modified version of the questionnaire was validated in a dermatology patient sample $(n=46)$ and presented high sensitivity and specificity $(100 \%$ and 92\% respectively) (Dufresne et al., 2001). Subsequently the BDDQ has been widely used for BDD screening, e.g. with 1000 dermatology and plastic surgery outpatients (Vulink et al., 2006), 100 psychiatric inpatients (Conroy et al., 2008), 160 patients with maxillofacial problems (Vulink et al., 2008) and 300 dermatological patients (Conrado et al., 2010).

The above-mentioned psychometric data are dependent on the study setting, including prevalence of the disease in the examined sample. The results of previous prevalence studies suggest that BDD rates vary significantly depending on the population studied, sample size, and assessment methods, which may be a result of methodological differences and limitations (e.g. non-representative populations, small sample sizes and insufficient assessment methods) (Buhlmann and Winter, 2011). To our knowledge, neither the BDDQ nor any other BDD assessment instruments, have been validated in the general population. In Scandinavia, there are no instruments available for screening of BDD that we know of. Presuming that the occurrence of, and undetected suffering from, BDD are at levels similar to other Western countries, translating a screening instrument into Swedish was justified. 
Aims

The purpose of this study was to translate the BDDQ into Swedish, and validate the translated version in a community sample. Since BDD seems to be slightly more common in women, a sample from the female population was initially chosen, while planning to subsequently validate the instrument also in men. 


\section{Methods}

\subsection{Translation}

Permission to use the BDDQ was given by K A Phillips. The questionnaire was translated into Swedish, using centered translation, i.e. verbatim. Centered translation was chosen based on the desirable features of the BDDQ, with a wording that closely resembles the diagnostic criteria in DSM-IV. The questionnaire was translated into Swedish by two of the authors (S Brohede and K Wijma), using the wording from the Swedish translation of DSM-IV where applicable. Two independent translators translated the BDDQ back into English. The backtranslation was subsequently compared with the original text. The process required three rounds of translation before the Swedish translation was concluded to be satisfactory by all the translators.

\subsection{Subjects}

The study sample comprised 7000 women aged 18-60 in the County of Östergötland, in the southeast of Sweden. Sampling randomization, out of women in the national population register, was made using the Statistical Package for Social Sciences (SPSS). Questionnaires were sent via mail to the selected women on Oct 1st 2009. Enclosed were a return envelope and a letter explaining that by returning a filled out questionnaire, consent to participation would be assumed. A follow-up reminder with a second copy of the questionnaire was sent to the 4700 women who had not responded after four weeks. The project was approved by the regional ethical review board for research (Dnr M103-09) and all the participants gave informed consent.

\subsection{Measures}

The questions and scoring of the BDDQ are presented in Table 1. Positive answers to the first two questions: "Are you very concerned about the appearance of some part(s) of your body that you consider especially unattractive?" and "Do these concerns preoccupy you? That is, do you think about them a lot and wish you could think about them less?" were required to continue the questionnaire. A positive answer to at least one part of the third question, assessing distress and impairment caused by the preoccupation, was further required for a BDD diagnosis. The fourth question: "How much time do you spend thinking about your defect(s) per day on average?" had the response alternatives a) less than 1 hour per day, b) 13 hours per day and c) $>3$ hours per day. When interpreting the BDDQ, Phillips suggests that the time taken up by thinking about the perceived defect should be at least one hour per day in order to fulfill the DSM-IV BDD diagnosis (Phillips, 1998). Thinking about the appearance flaw "at least an hour per day" is also a (optional) time-criterion when diagnosing BDD according to the Structured Clinical Interview for DSM-IV (SCID) (First et al., 2002). Therefore, positive answers to the first three questions of the BDDQ in combination with answer b) or c) on question four were required to fulfill the BDD criteria. The last question: "Is your main concern with your appearance that you aren't thin enough or that you might become fat?" was used to exclude people primarily concerned about not being thin enough, in order not to over-diagnose BDD when an eating disorder might be a more accurate diagnosis (Phillips, 1998). 
Table 1. Scoring of the Body Dysmorphic Disorder Questionnaire (BDDQ)

\begin{tabular}{|c|c|c|}
\hline BDDQ question & Answer $^{\mathrm{a}}$ & $\begin{array}{l}\text { BDDQ } \\
\text { score }^{b}\end{array}$ \\
\hline $\begin{array}{l}\text { 1. Are you very concerned about the apperance of some } \\
\text { part(s) of your body that you consider especially } \\
\text { unattractive? }\end{array}$ & Yes & 1 \\
\hline $\begin{array}{l}\text { 2. Do these concerns preoccupy you? That is, do you } \\
\text { think about them a lot and wish you could think about } \\
\text { them less? }\end{array}$ & Yes & 2 \\
\hline \multicolumn{3}{|l|}{$\begin{array}{l}3 \text { a. Has your defect(s) caused you a lot of distress, } \\
\text { torment or pain? }\end{array}$} \\
\hline $\begin{array}{l}\text { b. Has your defect(s) significantly interfered with your } \\
\text { social life? }\end{array}$ & \multirow{3}{*}{$\begin{array}{l}\text { Yes to at } \\
\text { least one } \\
\text { question }\end{array}$} & \multirow{3}{*}{3} \\
\hline $\begin{array}{l}\text { c. Has your defect(s) significantly interfered with your } \\
\text { school work, your job or your ability to function in } \\
\text { your role? }\end{array}$ & & \\
\hline d. Are there things you avoid because of your defect(s)? & & \\
\hline $\begin{array}{l}\text { 4. How much time do you spend thinking about your } \\
\text { defect(s) per day on average? }\end{array}$ & $\geq 1$ hour & 4 \\
\hline $\begin{array}{l}\text { 5. Is your main concern with your appearance that you } \\
\text { aren't thin enough or that you might become fat? }\end{array}$ & $\begin{array}{c}\text { Yes }= \\
\text { excluded }\end{array}$ & \\
\hline
\end{tabular}

$\overline{\bar{a}}$ Only if the answer is "yes", the subsequent question is asked.

${ }^{\mathrm{b}}$ Cumulative scoring. 4 points is considered a positive BDD-screening according to the BDDQ.

For the validation, the BDDQ was scored from 0 to 4 points (Table 1 ). The scoring from $0-4$ was made in order to evaluate the questionnaire's capacity to distinguish BDD from non-BDD at increasing levels of appearance concern. A BDDQ score of 4 was equal to the fulfillment of the BDD criteria and was thus considered a positive BDD-screening. The respondents were grouped into the five BDDQ score levels $0,1,2,3$ and 4 . To ensure a validation of the questionnaire's properties at the different levels of appearance concern, an interview sample was created that included respondents from all these BDDQ score levels. Twenty women were randomly selected from within each group with BDD scores of $0,1,2$ and 3 respectively. From the group with a BDDQ score of 4, 25 women were randomly selected. More women were selected from this group in order to assess the questionnaire's validity particularly at the critical cut-off at 4 points (i.e. positive BDD-screening). Randomization was carried out using www.random.org. These 105 women were all invited and 88 of them agreed to participate in diagnostic interviews at three hospitals in the County of Östergötland. 
2010. The interview followed a structured diagnostic method: the Structured Clinical Interview for DSM-IV-TR Axis I Disorders, Research Version, Non-patient Edition (SCID-I/NP) (First et al., 2002). SCID is a standard diagnostic instrument in psychiatry, and the SCID-I module for BDD assesses all three DSM-IV diagnostic criteria. The first author is a medical doctor, with experience in diagnosing BDD using the BDD diagnostic module, a SCID-like manual for BDD (Philips, 1998), in a previous study of women suffering from hirsutism (Brohede and co-workers, unpublished results). The last author, a clinical psychologist, was available for consultation when necessary. For a second opinion a psychiatrist, who was experienced in diagnosing BDD, could be consulted via telephone. As the physical defect must be nonexistent or slight to meet the diagnostic criteria, the women's perceived appearance flaws were assessed by the interviewer using a Likert-scale, similar to rating scales used in previous research (Dufresne et al., 2001; Conroy et al., 2008; Conrado et al., 2010). The scale ranged from 1 to $3 ; 1=$ no flaw present, $2=$ minimal $/$ slight flaw present, $3=$ flaw present and clearly noticeable within conversational distance. BDD was diagnosed if all the diagnostic criteria were met according to the SCID interview and the appearance flaw was rated 1 or 2 . The SCID interview, together with the clinical assessment of the perceived appearance flaw, was considered the gold standard for diagnosing BDD. Before the interview, the interviewer was blinded to the interviewees' scoring on the BDDQ. After the interview, the results from the BDDQ were compared to the SCID evaluation.

\subsection{Statistical analyses}

Demographic characteristics of the interview sample were compared with the total sample using Pearson's chi-square analysis (employing Fisher's exact test when indicated) for categorical variables and the Mann-Whitney U test for ordinal variables. P-value $<0.05$ was considered statistically significant in all analyses. Concurrent validity was assessed using sensitivity, specificity, positive and negative predictive value and likelihood ratio (Fletcher et al., 1996).

\section{Results}

\subsection{Demographic characteristics of the sample}

A total of 2891 women participated in the study. Excluding the 80 questionnaires that were returned because the addresses were unknown, the response rate was $42 \%$. Since the response rate was lower than expected, a representativeness analysis was carried out. The sample ( $n=2891$ ) was compared to the source population (women aged 18-60 in the County of Östergötland) using the socio-demographic data available from the respondents and corresponding data available from existing Swedish population statistics. These parameters were age, degree of education completed, employment status and occupation. The respondents did not differ significantly from the population in these parameters (Brohede and co-workers, unpublished results). 
Table 2. Demographic data of the interview sample compared with the total sample

\begin{tabular}{|c|c|c|c|}
\hline & $\begin{array}{c}\text { Total sample, } \% \\
\qquad(n=2891)\end{array}$ & $\begin{array}{c}\text { Interview sample, } \% \\
\qquad(\mathrm{n}=88)\end{array}$ & $\mathrm{P}$-value \\
\hline Age (years) & & & $0.716^{\mathrm{a}}$ \\
\hline $18-30$ & 29.2 & 27.6 & \\
\hline $31-45$ & 36.4 & 42.5 & \\
\hline $46-60$ & 34.4 & 29.9 & \\
\hline Education (highest completed) & & & $0.352^{\mathrm{b}}$ \\
\hline Elementary school & 8.9 & 8.0 & \\
\hline High school & 45.1 & 36.4 & \\
\hline University & 39.0 & 47.7 & \\
\hline Other & 7.1 & 8.0 & \\
\hline Marital status & & & $0.014^{\mathrm{b} *}$ \\
\hline Single & 17.4 & 26.1 & \\
\hline In a relationship (not cohabiting) & 9.4 & 14.8 & \\
\hline Cohabiting/Married & 72.9 & 59.1 & \\
\hline Occupation & & & $0.767^{\mathrm{b}}$ \\
\hline Employed & 67.8 & 69.3 & \\
\hline Leave of absence/Parental leave & 4.9 & 2.3 & \\
\hline Unemployed & 5.9 & 4.5 & \\
\hline Student & 13.3 & 13.6 & \\
\hline Sick leave/Disability pension/Social support & 6.5 & 9.1 & \\
\hline Other & 1.6 & 1.1 & \\
\hline Household income (Swedish crowns/month) & & & $0.392^{\mathrm{a}}$ \\
\hline$<10000$ & 10.4 & 10.2 & \\
\hline $10000-29999$ & 28.9 & 36.9 & \\
\hline $30000-49999$ & 34.0 & 26.1 & \\
\hline$>50000$ & 26.6 & 26.1 & \\
\hline
\end{tabular}

${ }^{\mathrm{a}}$ Mann Whitney U-test.

${ }^{\mathrm{b}}$ Pearson's chi square test.

$* \mathrm{p}<0.05$ i.e. statistically significant difference.

One hundred five women were invited for diagnostic interviews and 88 of these women agreed to participate. Table 2 presents the demographic characteristics of the interview sample $(n=88)$ compared to the total sample $(n=2891)$. There were no statistically significant differences between the two samples regarding age, educational level, occupation and household income. Statistically significant differences were found in marital status. In the 
interview sample, $26 \%$ of the women were single, compared to $17 \%$ in the total sample, whereas 59\% were cohabiting/married, compared to $73 \%$ in the total sample.

\subsection{Concurrent validity}

The interviewed women's BDDQ scores and BDD diagnoses are presented in Table 3. A BDDQ score of 4 was considered a positive BDD-screening $(n=24)$. Of these 24 women, 17 were diagnosed with BDD according to the SCID and clinical assessment. Of the seven women who were not diagnosed with BDD, six women did not meet the BDD criteria according to the SCID and one woman had a defect severity rating of 3. One woman who had a negative BDDscreening (BDDQ score 3) was diagnosed with BDD, since BDD criteria were met according to the SCID interview and she had a defect severity rating of 2 .

According to preliminary results, the prevalence of BDDQ-positive women in the total sample was similar to BDD prevalence rates found in previous studies of community samples. The proportion of those with a BDDQ score of 4 in the interview sample was 24/88 (27\%), and thus was higher in comparison to the total sample. Similarly, the proportion of those with a BDDQ score of 3 was also higher in the interview sample compared to the total sample.

Table 3. Number of women with different scores on the Body Dysmorphic Disorder Questionnaire (BDDQ) and their diagnosis according to the SCID evaluation together with clinical assessment $(n=88)$

\begin{tabular}{cllc}
\hline \hline BDDQ score & BDD & No BDD & Total \\
\hline 0 & $0(0 \%)$ & $20(100 \%)$ & 20 \\
1 & $0(0 \%)$ & $18(100 \%)$ & 18 \\
2 & $0(0 \%)$ & $11(100 \%)$ & 11 \\
3 & $1(7 \%)$ & $14(93 \%)$ & 15 \\
4 & $17(71 \%)$ & $7(29 \%)$ & 24 \\
\hline \hline
\end{tabular}

Note. SCID=Structured Clinical Interview for DSM-IV.

Table 4 summarizes the validation results. In this study, sensitivity is defined as the proportion of women with BDD determined by the SCID and clinical assessment, who screened positive for BDD using the BDDQ. Specificity is defined as the proportion of women without BDD according to the SCID and clinical assessment, who screened negative for BDD using the BDDQ. The positive predictive value expresses the probability of having BDD according to the SCID and clinical assessment when BDDQ-screening is positive, while the negative predictive value is the probability of not having BDD when BDDQ-screening is negative. The likelihood ratio summarizes sensitivity and specificity and is expressed as odds. The (positive) likelihood ratio expresses how many times more likely it is that BDDQscreening will be positive in women with BDD compared to in women without BDD. The advantages of likelihood ratio are that the test validity is expressed with only one figure and is 
more independent of prevalence rates (Fletcher et al., 1996). The Swedish version of the BDDQ displayed high rates of sensitivity (94\%) and specificity (90\%) in the community-based sample of women. The obtained (positive) likelihood ratio was 9.4. The positive predictive value in this sample was $71 \%$ and the negative predictive value $98 \%$.

Table 4. Validation of the Body Dysmorphic Disorder Questionnaire (BDDQ) compared to the Structured Clinical Interview for DSM-IV (SCID) together with clinical assessment as the gold standard $(n=88)$

\begin{tabular}{lcc}
\hline \hline & \multicolumn{2}{c}{ SCID evaluation (gold standard) } \\
& BDD & No BDD \\
\hline BDDQ positive & $17^{\mathrm{a}}$ & $7^{\mathrm{b}}$ \\
BDDQ negative & $1^{\mathrm{c}}$ & $63^{\mathrm{d}}$ \\
\hline & & \\
Validation concept & Value (95\% confidence interval) \\
\hline Sensitivity $(a /(a+c))$ & $94 \%(71-100)$ \\
Specificity $(d /(b+d))$ & $90 \%(80-96)$ \\
Positive predictive value $(a /(a+b))$ & $71 \%(49-87)$ \\
Negative predictive value $(d /(c+d))$ & $98 \%(90-100)$ \\
Likelihood ratio $(a /(a+c) /(b /(b+d))$ & $9.4(4.6-19.2)$ \\
\hline \hline
\end{tabular}

$\overline{\mathrm{a}, \mathrm{b}, \mathrm{c}, \mathrm{d}}$ Letters are used to illustrate the calculated values of the different validation concepts.

Note. The sample had been selected to increase the proportion of BDDQ-positive women, in order to evaluate the questionnaire's properties at the cut-off point. 


\section{Discussion}

To our knowledge, the Swedish version of the Body Dysmorphic Disorder Questionnaire is the first screening instrument for BDD in a Scandinavian language. Moreover, this is the first validation of a BDD assessment instrument in a community sample that we know of. The BDDQ was validated against a face-to-face diagnostic interview using the SCID together with a clinical assessment of the appearance flaw as the gold standard. The proportion of women with positive BDD-screening was increased for methodological reasons, reaching a prevalence of $27 \%$. With a specificity of $94 \%$, a specificity of $90 \%$ and a likelihood ratio of 9.4 , the BDDQ proved to have good concurrent validity in this subsample.

Although the response rate does not necessarily affect the quality of the validation, a representativeness analysis was performed, since the response rate was lower than expected (42\%). There were no statistically significant differences between the total sample $(n=2891)$ and the source population (women aged 18-60 in the County of Östergötland, Sweden) regarding demographic characteristics available from existing Swedish population statistics. The interview sample $(n=88)$ was representative of the total sample concerning age, educational level, occupation, and household income. The women in the interview sample were single to a higher degree and cohabiting/married to a lesser degree than in the total sample. As the prevalence of BDD was higher in the interview sample compared to the total sample, at least part of the explanation is probably that women with BDD are more likely to be single. Previous large population studies have reported that individuals with BDD are more frequently divorced or single than individuals without BDD (Rief et al., 2006; Koran et al., 2008). This is not surprising, since the disorder often leads to impaired functioning in relationships, socializing and intimacy (Phillips, 1991).

The high sensitivity and specificity obtained are comparable to previous research. The BDDQ has previously been validated in a few studies. In two different psychiatric inpatient samples, $\mathrm{n}=66$ (Phillips et al., 1995) and $\mathrm{n}=122$ (Grant et al., 2001), the instrument displayed a sensitivity of $100 \%$ and a specificity of $89 \%$ and $93 \%$ respectively. In a dermatology patient sample $(n=46)$, a modified version of BDDQ had a sensitivity of $100 \%$ and specificity of $92 \%$ (Dufresne et al., 2001). The specificity of $90 \%$ shown in the present study falls in the range of earlier validation results and confirms the specificity of the instrument, also when used in a community based sample. The sensitivity was lower (94\%) than in previous validation studies, which can be explained by the different setting with a sample from the general population rather than psychiatric/dermatologic settings. Another explanation may be the different methodological approaches. In our study we used face-to-face assessment with the SCID interview as the gold standard for diagnosing BDD, whereas other researchers, e.g. Dufresne et al (2001) used the Body Dysmorphic Disorder Diagnostic Module, a clinicianadministered semi-structured instrument, as the gold standard.

In addition to presenting high values of sensitivity and specificity, the BDDQ also had a very high positive predictive value of $98 \%$, whereas the negative predictive value was $71 \%$ in this sample. The predictive values depend critically on the prevalence of the disorder in the population concerned (Altman and Bland, 1994, Fletcher et al., 1996). The interview sample was selected in order to evaluate the questionnaire's properties at the cut-off point (4 points on BDDQ to screen positive for BDD), and therefore the proportion of women with a positive BDD-screening was higher compared to the total sample. If 100 women had been randomly 
selected for interview from the total sample, it is likely that only a small percentage would have screened positive for BDD, and no proper assessment of the cut-off at 4 points could have been made. Since there were limitations to the number of women that we could involve in the validation procedure, the increased proportions of scores 3 and 4 made the assessment of the questionnaire's properties at the critical cut-of point more thorough. The limitations were mainly due to the choice of face-to-face diagnostic interviews, which is indeed the gold standard for diagnosing BDD (Phillips, 2009). However, the procedure for increasing the prevalence of positive BDDQ in the interview sample does affect the validation properties of the test, especially the positive and negative predictive values. Though the present validation figures are indeed assessed in a population-based sample, they vary in populations with different prevalence of BDD. The positive predictive value of the questionnaire would presumably decrease and the negative predictive value increase in a population with a lower prevalence of BDD (Fletcher et al., 1996). Nevertheless, the BDDQ also presented a high likelihood ratio, which is less dependent on prevalence rates. A likelihood ratio of 9.4 means that it is almost ten times more likely that a positive BDDQ will be found in women with BDD than in women without BDD.

The ability of the BDDQ to differentiate between BDD and eating disorders was not explored, which is a weakness of the study. By excluding from BDD diagnosis all who were concerned about not being thin enough or who were afraid of becoming fat, there is a risk of underdiagnosing BDD. There are several reasons for the chosen approach. When developing BDDQ, Phillips stated that in screening for BDD a "yes" answer to question 5 (Table 1) might indicate the presence of an eating disorder rather than BDD (Phillips, 1998). Since we excluded these women from the validation, no clinical assessment of their perceived appearance defect was conducted. Women, who are indeed overweight or obese and preoccupied by this, might also reply "yes" to this question, even if they were not suffering from an eating disorder. Rief et al (2006) also excluded all individuals from BDD diagnosis, who reported their body weight as the primary cause of their appearance concern. In the other large German population study, a more specific way of excluding eating disorder was used (Buhlmann et al., 2010). Additional questions were asked to exclude individuals who reported their body weight as the main focus of concern, and one of the following symptoms in the past three months were necessary for BDD exclusion: repeatedly fasting for at least $24 \mathrm{~h}$, repeatedly making oneself vomit after eating meals, or repeatedly taking more than twice the recommended amount of diuretics. Nevertheless, BDD has been reported to be comorbid with eating disorders to a great extent. Indeed, 32.5\% of 200 BDD-patients met the criteria for a lifetime comorbid eating disorder (Phillips et al., 2006) and correspondingly, in patients with an eating disorder 39\% (Grant et al., 2002) and 45\% (Dingemans et al., 2012) also met the criteria for BDD. The possibility of comorbidity must therefore be acknowledged. Undoubtedly, further research is necessary regarding this predicament when screening for BDD.

There are some other limitations to the present study. It is an obvious limitation that the study only included women. There is a need for validated BDD assessment instruments also for men, since the disorder seems to be almost as common in men, with a gender ratio of females to males of 1:1 to 3:2 (Phillips et al., 2008). However, for practical and economical reasons we did not have the possibility to extend our validation study to also include men. Since BDD seems to be slightly more common in women, we chose to initially focus on women. However, the Swedish translation of BDDQ needs to be validated also in men. To assess the semantic equivalence of the Swedish BDDQ compared to the original, both versions should ideally have been evaluated by bilingual test persons and agreement measured. Prior 
to the larger study, the BDDQ could also have been tested in a smaller sample of the Swedish population, to ensure a "culture validation". The decision not to perform a pre-test was based on the assumption that BDD has such a low prevalence that a very large test sample would be needed to ensure that at least some study participants with BDD would be included. The reliability of the BDDQ could have been determined by test/re-test to assess the stability of the instrument. The same respondent would then fill out the BDDQ on two different occasions, the results would be compared, and correlations computed. Letting the study participants fill out another BDDQ in connection with the diagnostic interview was considered. However, the BDDQ and the SCID instruments are both derived from, and closely resemble, the DSM-IV criteria, and therefore use very similar wording. The risk of the BDDQ influencing the respondents' answers in the SCID interview (or vice versa, depending on which was first) was presumed to be considerable and not worth taking, if it would impair the validation accuracy. The diagnostic interview using the SCID was carried out by a clinician, who had some experience in diagnosing BDD using a structured interview manual, but was nevertheless not a psychiatrist. Involving instead at least two assessors, preferably experienced psychiatrists, would have allowed us to evaluate interrater reliability.

In spite of these limitations, our study provides some valuable new knowledge. The BDDQ was valid in the community-based sample of women. The high likelihood ratio indicates that the instrument can be used in general female populations with different expected prevalence of BDD. The BDDQ can probably be of value also when screening for BDD in female patients in settings where the prevalence of BDD can be assumed to be higher than in the general population, for example in clinics for primary care, dermatology and plastic surgery. This is important, as people with BDD often go undiagnosed in clinical practice (Zimmerman and Mattia, 1998; Grant et al., 2001; Phillips and Hollander, 2008). 


\section{References}

Altman, D.G., Bland, M.J., 1994. Statistics Notes: Diagnostic tests 2: predictive values. British Medical Journal 309,102.1.

American Psychiatric Association, 2000. Diagnostic and statistical manual of mental disorders : DSM-IV-TR, Washington, DC, American Psychiatric Association.

Bienvenu, O.J., Samuels, J.F., Riddle, M.A., Hoehn-Saric, R., Liang, K.Y., Cullen, B.A., Grados, M.A., Nestadt, G., 2000. The relationship of obsessive-compulsive disorder to possible spectrum disorders: results from a family study. Biological Psychiatry 48, 287-293.

Bowe, W.P., Leyden, J.J., Crerand, C.E., Sarwer, D.B., Margolis, D.J., 2007. Body dysmorphic disorder symptoms among patients with acne vulgaris. Journal of the American Academy of Dermatology 57, 222-230.

Buhlmann, U., Glaesmer, H., Mewes, R., Fama, J.M., Wilhelm, S., Brähler, E., Rief, W., 2010. Updates on the prevalence of body dysmorphic disorder: A population-based survey. Psychiatry Research 178, 171-175.

Buhlmann, U., Winter, A., 2011. Perceived ugliness: an update on treatment-relevant aspects of body dysmorphic disorder. Current Psychiatry Reports 13, 283-288.

Conrado, L.A., Hounie, A.G., Diniz, J.B., Fossaluza, V., Torres, A.R., Miguel, E.C., Rivitti, E.A., 2010. Body dysmorphic disorder among dermatologic patients: Prevalence and clinical features. Journal of American Academy of Dermatology 63, 235-243.

Conroy, M., Menard, W., Fleming-Ives, K., Modha, P., Cerullo, H., Phillips, K.A., 2008. Prevalence and clinical characteristics of body dysmorphic disorder in an adult inpatient setting. General Hospital Psychiatry 30, 67-72.

Crerand, C.E., Phillips, K.A., Menard, W., Fay, C., 2005. Nonpsychiatric medical treatment of body dysmorphic disorder. Psychosomatics 46, 549-555.

Dingemans, A.E., Van Rood, Y.R., De Groot, I., Van Furth, E.F., 2012. Body dysmorphic disorder in patients with an eating disorder: prevalence and characteristics. The International Journal of Eating Disorders 45, 562-569.

Dufresne, R.G., Phillips, K.A., Vittorio, C.C., Wilkel, C.S., 2001. A screening questionnaire for body dysmorphic disorder in a cosmetic dermatologic surgery practice. Dermatologic surgery : official publication for American Society for Dermatologic Surgery [et al.] 27, 457-462.

Faravelli, C., Salvatori, S., Galassi, F., Aiazzi, L., Drei, C., Cabras, P., 1997. Epidemiology of somatoform disorders: a community survey in Florence. Social Psychiatry and Psychiatric Epidemiology 32, 24-29.

First, M.B., Spitzer, R.L., Gibbon, M., Williams, J.B.W., 2002. Structured Clinical Interview for DSM-IV-TR Axis I Disorders, Research Version, Non-patient Edition. (SCID-I/NP). New York: Biometrics Research: New York State Psychiatric Institute.

Fletcher, R.H., Fletcher, S.W., Wagner, E.H., 1996. Clinical epidemiology. Baltimore, Williams \& Wilkins.

Grant, J.E., Kim, S.W., Crow, S.J., 2001. Prevalence and clinical features of body dysmorphic disorder in adolescent and adult psychiatric inpatients. The Journal of Clinical Psychiatry 62, 517-522. 
Grant, J.E., Kim, S.W., Eckert, E.D., 2002. Body dysmorphic disorder in patients with anorexia nervosa: prevalence, clinical features, and delusionality of body image. The International Journal of Eating Disorders 32, 291-300.

Kollei, I., Martin, A., Rein, K., Rotter, A., Jacobi, A., Mueller, A., 2011. Prevalence of body dysmorphic disorder in a German psychiatric inpatient sample. Psychiatry Research $189,153-155$.

Koran, L.M., Abujaoude, E., Large, M.D., Serpe, R.T., 2008. The prevalence of body dysmorphic disorder in the United States adult population. CNS Spectrums 13, 316-322.

Otto, M.W., Wilhelm, S., Cohen, L.S., Harlow, B.L., 2001. Prevalence of body dysmorphic disorder in a community sample of women. The American Journal of Psychiatry 158, 2061-2063.

Pavan, C., Simonato, P., Marini, M., Mazzoleni, F., Pavan, L., Vindigni, V., 2008. Psychopathologic aspects of body dysmorphic disorder: a literature review. Aesthetic Plastic Surgery 32, 473-484.

Phillips, K., 1998. The broken mirror : understanding and treating body dysmorphic disorder, Oxford, Oxford University Press.

Phillips, K.A., 1991. Body dysmorphic disorder: the distress of imagined ugliness. The American Journal of Psychiatry 148, 1138-1149.

Phillips, K.A., 2007. Suicidality in Body Dysmorphic Disorder. Primary Psychiatry 14, 58-66.

Phillips, K.A., 2009. Understanding body dysmorphic disorder: an essential guide, Oxford, Oxford University Press.

Phillips, K.A., Atala, K.D., Pope, H.G., 1995. Diagnostic instruments for body dysmorphic disorder. New Research Program and Abstracts, American Psychiatric Association 148th annual meeting. Miami: American Psychiatric Association.

Phillips, K.A., Didie, E.R., Feusner, J., Wilhelm, S., 2008. Body dysmorphic disorder: treating an underrecognized disorder. The American Journal of Psychiatry 165, 1111-1118.

Phillips, K.A., Dufresne, R.G. Jr., Wilkel, C.S., Vittorio, C.C., 2000. Rate of body dysmorphic disorder in dermatology patients. Journal of the American Academy of Dermatology 42, 436-441.

Phillips, K.A., Grant, J., Siniscalchi, J., Albertini, R.S., 2001. Surgical and nonpsychiatric medical treatment of patients with body dysmorphic disorder. Psychosomatics 42, 504-510.

Phillips, K.A., Hollander, E., 2008. Treating body dysmorphic disorder with medication: evidence, misconceptions, and a suggested approach. Body Image 5, 13-27.

Phillips, K.A., Menard, W., 2006. Suicidality in body dysmorphic disorder: a prospective study. The American Journal of Psychiatry 163, 1280-1282.

Phillips, K.A., Menard, W., Fay, C., 2006. Gender similarities and differences in 200 individuals with body dysmorphic disorder. Comprehensive Psychiatry 47, 77-87.

Rief, W., Buhlmann, U., Wilhelm, S., Borkenhagen, A., Brahler, E., 2006. The prevalence of body dysmorphic disorder: a population-based survey. Psychological Medicine 36, 877-885.

Uzun, O., Basoglu, C., Akar, A., Cansever, A., Ozsahin, A., Cetin, M., Ebrinc, S., 2003. Body dysmorphic disorder in patients with acne. Comprehensive Psychiatry 44, 415-419. 
Vulink, N.C., Rosenberg, A., Plooij, J.M., Koole, R., Berge, S.J., Denys, D., 2008. Body dysmorphic disorder screening in maxillofacial outpatients presenting for orthognathic surgery. International Journal of Oral and Maxillofacial Surgery 37, 985-991.

Vulink, N.C., Sigurdsson, V., Kon, M., Bruijnzeel-Koomen, C.A., Westenberg, H.G., Denys, D., 2006. [Body dysmorphic disorder in 3-8\% of patients in outpatient dermatology and plastic surgery clinics]. Nederlands Tijdschrift voor Eneeskunde 150, 97-100.

Zimmerman, M., Mattia, J.I., 1998. Body dysmorphic disorder in psychiatric outpatients: recognition, prevalence, comorbidity, demographic, and clinical correlates. Comprehensive Psychiatry 39, 265-270. 